\title{
Identification of pivotal IncRNAs in papillary thyroid cancer using LncRNA-mRNA-miRNA ceRNA network analysis
}

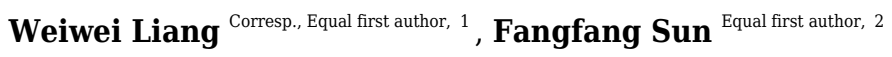 \\ ${ }^{1}$ Department of Endocrinology, The Second Affiliated Hospital, Zhejiang University School of Medicine, Hangzhou, China \\ Key Laboratory of Cancer Prevention and Interve
University School of Medicine, Hangzhou, China \\ Corresponding Author: Weiwei Liang \\ Email address: helenliangww@zju.edu.cn
}

Background To identify pivotal IncRNAs in papillary thyroid cancer (PTC) using IncRNA-mRNA-miRNA ceRNA network analysis.

Methods We obtained gene expression profiles from the Gene Expression Omnibus database. Cancer specific IncRNA, cancer specific miRNA and cancer specific mRNA were identified. An integrated analysis was conducted to detect potential IncRNA-miRNA-mRNA ceRNA in regulating disease transformation. The IncRNA regulated $\mathrm{GO}$ terms and regulated pathways were performed by function analysis. Survival analysis was performed for the pivotal IncRNAs.

Results A total of 4 IncRNAs, 15 miRNAs and 375 mRNAs are identified as the key mediators in the pathophysiological processes of PTC. Gene Ontology annotation enrichment analysis showed the most relevant $\mathrm{GO}$ terms are signal transduction, integral component of membrane and calcium ion binding. KEGG pathway enrichment analysis showed DEGs mainly enriched in pathways in cancer, PI3K-Akt signaling pathway and focal adhesion. Among 4 IncRNAs, only SLC26A4-AS1 was significantly associated with PTC patient disease free survival.

Conclusion This study has constructed IncRNA-mRNA-miRNA ceRNA networks in PTC. The study provides a set of pivotal IncRNAs for future investigation into the molecular mechanisms. 


\section{Identification Pivotal IncRNAs in Papillary Thyroid Cancer Using}

2 LncRNA-mRNA-miRNA ceRNA Network Analysis Running title:

3 ceRNA Network of Thyroid Cancer

4

5 Weiwei Liang ${ }^{1}$, FangFang Sun ${ }^{2}$

$6{ }^{1}$ Department of Endocrinology, The Second Affiliated Hospital, Zhejiang University School of Medicine, Hangzhou,

7 P. R. China. Mail: helenliangww@zju.edu.cn

$8 \quad{ }^{2}$ Key Laboratory of Cancer Prevention and Intervention, China National Ministry of Education, The Second Affiliated

9 Hospital, Cancer Institute, Zhejiang University School of Medicine, Hangzhou, P. R. China. Mail: sun77@zju.edu.cn

\section{CORRESPONDENCE:}

13 Dr. Weiwei Liang

14 1Department of Endocrinology, The Second Affiliated Hospital, Zhejiang University School of Medicine, Hangzhou,

15 P. R. China. Mail: helenliangww@zju.edu.cn

16 Liang and Sun contributed equally to this work and should be considered as first co-authors. 


\section{Abstract}

\section{Background}

To identify pivotal lncRNAs in papillary thyroid cancer (PTC) using lncRNA-mRNA-miRNA ceRNA network 25 analysis.

\section{Methods}

We obtained gene expression profiles from the Gene Expression Omnibus database. Cancer specific lncRNA, 28 cancer specific miRNA and cancer specific mRNA were identified. An integrated analysis was conducted to detect 29 potential lncRNA-miRNA-mRNA ceRNA in regulating disease transformation. The lncRNA regulated GO terms and 30 regulated pathways were performed by function analysis. Survival analysis was performed for the pivotal lncRNAs.

\section{Results}

A total of 4 lncRNAs, 15 miRNAs and 375 mRNAs are identified as the key mediators in the pathophysiological 33 processes of PTC. Gene Ontology annotation enrichment analysis showed the most relevant GO terms are signal 34 transduction, integral component of membrane and calcium ion binding. KEGG pathway enrichment analysis showed 35 DEGs mainly enriched in pathways in cancer, PI3K-Akt signaling pathway and focal adhesion. Among 4 lncRNAs, 36 only SLC26A4-AS1 was significantly associated with PTC patient disease free survival.

\section{Conclusion}

38 This study has constructed IncRNA-mRNA-miRNA ceRNA networks in PTC. The study provides a set of pivotal 39 IncRNAs for future investigation into the molecular mechanisms.

41 Key words: IncRNA, ceRNA Network, Papillary Thyroid Cancer 


\section{Introduction}

Most of the human genome transcribes RNAs that do not code for proteins. These non-coding RNAs (ncRNAs) affect normal expression of the genes, including oncogenes and tumour suppressive genes, which make them a new class of targets for study in tumorigenesis. NcRNAs include microRNA(miRNA), long non-coding RNA(lncRNA), circular RNA et al. MiRNA are highly conserved non-coding RNAs of approximately 21-24 nucleotide, which can interact with target mRNAs to regulate the expression of the gene. Studies have shown that miRNAs are involved in the initiation and progression of cancers[1], and miRNA-targeted therapeutics have already reached clinical development[2]. Recently, the importance of IncRNA is gradually realized. LncRNA is a group of non-coding RNAs (IncRNAs) that are more than 200 base pairs. LncRNAs have diverse role in genetic regulation. Recent studies have demonstrated that lncRNA play a major regulatory role in tumorigenesis[3].

The competing endogenous RNA (ceRNA) hypothesis, presented by Salmena et al.[4], has been described as the

"Rosetta Stone" for decoding the RNA language used in regulating RNA crosstalk and modulating biological functions. RNAs regulate each other with miRNAs response elements (MREs) and this mechanism is known as "competing endogenous RNA (ceRNA)" hypothesis. Many studies have suggested miRNA-mediated ceRNA regulatory mechanisms play crucial roles in the initiation and development of tumors[5].

Papillary thyroid cancer (PTC) is the most common type of thyroid cancer. PTC accounts for about $80 \%$ of all thyroid cancers[6]. It is the fifth most common cancer in women. The incidence of thyroid cancer in the United States and worldwide has increased $300 \%$ over the past 3 decades, due predominantly to an increase in PTC[7]. One study predicts that PTC will become the third most common cancer in women at a cost of \$19-21 billion in the United States in 2019[8]. Although PTC generally has a good prognosis, 10\% patients suffer from local recurrence and/or distant metastasis. Thus, it is essential to clarify the mechanisms of PTC and identify latent biomarkers. Recently, there is an increasing number of lncRNAs discovered in tumorigenesis of PTC[9,10]. Feng et al. found lncRNA n384546 promotes thyroid papillary cancer progression and metastasis[11]. Liang et al.'s recent study showed LncRNA MCM3AP-AS1 promotes proliferation and invasion through regulating miR-211-5p/SPARC axis in papillary thyroid cancer[12]. But most of the researches focus on one particular lncRNA, our knowledge about the lncRNA-mRNAmiRNA ceRNA network of PTC is still limited.

In this study, we have taken full advantage of the rich data from the GEO database. The aberrant expression profiles of IncRNA, mRNA, and miRNA in PTC were filtered. The lncRN-miRNA-mRNA ceRNA network was 


\section{MATERIALS AND METHODS}

77

\section{Data sources}

We conducted a search of Gene Expression Omnibus (GEO) (www.ncbi.nlm.nih.gov/geo/) for high-throughput genomics experiments of papillary thyroid carcinoma. We used the following search terms: thyroid tumor, thyroid carcinoma, papillary thyroid carcinoma, papillary carcinoma of thyroid. The RNA sequencing expression data of 3 datasets (GSE33630, GSE3467, GSE3678) were obtained from GEO. DESeq2 package[13] was used to identify different expressed genes. The quality of gene expression data was analyzed and visualized using the ggplot2 package of R software for each group and sample.

\section{Identification of cancer specific IncRNA, cancer specific miRNAs, cancer specific mRNA}

The analysis of differentially expressed genes was carried out by limma package[14], which includes lmFit, eBayes, and topTable functions. $\mathrm{P}<0.05$ and $\operatorname{abs}(\log 2)$ fold change $(\mathrm{FC})>1$ were used as the cut-off criteria. Commonly different changed genes(DEGs) from the datasets were integrated using Venn analysis. LncRNA, mRNA and miRNA were identified by bioMart package[15].

\section{Construction of IncRNA-miRNA-mRNA network of PTC}

The lncRNA-miRNA-mRNA network of each cancer was constructed by following steps:

1. Prediction of miRNA targeted lncRNA: we used DIANA-LncBase v2 (http://carolina.imis.athenainnovation.gr/)[16], starBase (http://starbase.sysu.edu.cn/) [17,18] and RAID v2.0 (http://www.rnasociety.org/raid/)[19] to analyze interactions between lncRNAs and miRNAs;

2. Identification of miRNA targeted mRNA: we used DIANA-TarBase v8 (http://carolina.imis.athenainnovation.gr/)[20] and TargetScan (http://www.targetscan.org/)[21] to predict miRNA-targeted mRNA; and

3. LncRNA-miRNA-mRNA network construction and visualization: Cytoscape 3.7.0 software[22] was utilized to construct and visualize ceRNA network as well as its subnetwork based on individual lncRNA.

\section{Functional analysis}

Gene ontology analysis (GO) was used to identify characteristic biological attributes. Kyoto Encyclopedia of Genes and Genomes pathway (KEGG)[23] enrichment analysis was performed to identify functional attributes. We use Search Tool for the Retrieval of Interacting Gene (STRING)[24], ClueGo[25] and Cluepedia[26] to access GO and pathway enrichment analysis. $\mathrm{p}<0.05$ was set as the cut-off criterion. 


\section{Survival analysis of pivotal IncRNAs}

The IncRNAs in PTC IncRNA-miRNA-mRNA network were indentified as pivotal IncRNAs. LncRNAs correlations with patient survival were featured in GEPIA[27]. Available TCGA patient survival data were used for Kaplan-Meier survival analysis and to generate overall and disease-free survival plots.

110

\section{RESULTS}

1123 GEO datasets (GSE3678, GSE3467, GSE33630) were obtained in our study: Three datasets were all based on 113 GPL570 platform (Affymetrix Human Genome U133 Plus 2.0 Array). GSE3678 dataset had 7 PTC samples and 7

114 paired normal thyroid tissue samples. GSE3467 included 9 PTC samples and 9 paired normal thyroid tissue samples.

115 GSE33630 had 49 PTC samples and 45 normal thyroid tissue samples.

116 The normalized expression values (ranging from 0 to $20 \log 2(\mathrm{TPM}+1)$ ) and the distributions were similar

117 between the three groups in each dataset (Fig. 1). Principal component showed that samples were easily grouped into

118 different groups in each dataset (Fig. 1). Based on these data distribution analyses, further bioinformatics analyses

119 could be performed based on the available data. Following data processing using LIMMA, 1189, 1194 and 2226

120 differentially expressed genes from the expression profile datasets GSE3678, GSE3467, GSE33630 were extracted

121 respectively (Fig. 2 A-D). 615 consistently expressed genes (sup. Table 1), including 4 lncRNAs, were identified by

122 Venn analysis (Fig. 2D). Hierarchical clustering of the identified DEGs is displayed as a heatmap in Fig. 2F.

123 The relationships between cancer specific lncRNAs and cancer specific miRNAs were first evaluated. We 124 obtained 96 abnormally expressed miRNAs significantly relevant to PTC survival from OncomiR. 4 PTC-specific 125 IncRNAs putatively interacted with 15 PTC-specific miRNAs. Then, the relationships between cancer specific 126 miRNAs and cancer specific mRNAs were evaluated. 375 PTC-specific mRNA targeted by 15 PTC-specific miRNAs 127 were identified. Based on the above data, the IncRNA-miRNA-mRNA ceRNA network of PTC (Fig. 3) was 128 established and plotted using Cytoscape 3.7.0.

129 We analyzed the mRNAs of the lncRNA-miRNA-mRNA ceRNA network and identified the lncRNA regulated

130 GO terms (Fig. 4, sup. Table 2-4). In PTC IncRNA-miRNA-mRNA ceRNA network, the result showed that the 131 mRNAs related to biological process(BP) were most relevant to signal transduction. mRNAs related to cellular 132 component (CC) were most relevant to integral component of membrane. mRNAs related to molecular function (MF) 133 were most relevant to calcium ion binding.

134 We performed pathway enrichment analysis of the mRNAs of the IncRNA-miRNA-mRNA ceRNA network 
135 and identified the lncRNA regulated pathway (Fig. 5, sup. Table 5). Functional analysis showed the top 3 lncRNA

136 regulated pathway are pathways in cancer, PI3K-Akt signaling pathway, focal adhesion.

137 We used GEPIA to perform survival analysis of pivotal lncRNAs (Fig. 6). We generated SLC26A4-AS1, NR2F1-

138 AS1 and TNRC6C-AS1's data with patients' survival information. Only SLC26A4-AS1 was significantly associated

139 with PTC patient disease free survival $(\mathrm{p}=0.002)$.

140

\section{DISCUSSION}

142 The last few years have nominated ceRNA hypothesis as a novel layer of gene regulation. Our knowledge about

143 the molecular mechanism of lncRNA in cancer is still limited. In the present study, we identified cancer specific

144 lncRNAs, miRNAs and mRNAs in PTC. According to the bioinformatics differential analysis we constructed a

145 ceRNA network. We predicted functions of differentially expressed genes in PTC by GO and pathway analysis.

146 Pivotal lncRNAs (SLC26A4-AS1, NR2F1-AS1, TNRC6C-AS1, LOC646736) from ceRNA network were further

147 investigated their correlations with survival based on RNA sequencing profile from TCGA.

148 Of the genes that were involved in ceRNA network, KEGG analysis showed that these genes were mainly 149 enriched in two pathways related with cancer: pathways in cancer and PI3K-Akt signaling pathway. There was 150 evidence showed that PI3K-Akt signaling pathway plays an important role in PTC tumorigenesis[28]. Hao et al.'s 151 study showed PI3K-Akt signaling pathway activation was associated with PTC cell proliferation, migration, and 152 invasion[29]. Zheng et al found TEKT4 promoted PTC cell metastasis through activating PI3K/Akt pathway[30]. The 153 regulation function of lncRNAs through PI3K/Akt pathway in PTC is worth study. There were only two studies 154 published focused on the regulation function of lncRNAs through PI3K/Akt pathway in PTC. Wen et al. found 155 lncRNA ABHD11-AS1 promotes tumour progression in PTC by regulating PI3K/AKT signalling pathway[31]. Liu's 156 study showed downregulation of NEAT1 reversed the radioactive iodine resistance of PTC cell via miR-101-

$1573 \mathrm{p} / \mathrm{FN} 1 / \mathrm{PI} 3 \mathrm{~K}-\mathrm{AKT}$ signaling pathway[32]. The ceRNA network identified in our study provided useful clues of 158 further study.

159 Among 4 pivotal lncRNAs, SLC26A4-AS1 is associated with PTC disease free survival. Fagerberg et al.'s study 160 showed SLC26A4-AS1 is restricted expressed in thyroid[33]. Our understanding of SLC26A4-AS1's function is 161 limited. Further study is worthwhile to detect SLC26A4-AS1's role in PTC.

162 LncRNA NR2F1-AS1 is broadly expressed in brain, gall bladder and other tissues. There is some evidence indicating 163 that LncRNA NR2F1-AS1 are associated with other cancer types. Huang et al.'s study showed lncRNA NR2F1-AS1 164 regulates hepatocellular carcinoma oxaliplatin resistance via miR-363[34]. Wang et al.'s recent study showed 
165 LncRNA NR2F1-AS1 is involved in the progression of endometrial cancer[35]. Studies of lncRNA NR2F1-AS1 in

166 PTC is limited, so they are worthy of future research.

167 TNRC6C-AS1 is broadly expressed in lymph node, spleen and other tissues. Hou's studies showed that lncRNA

168 TNRC6C-AS1 regulates UNC5B in thyroid cancer to influence cell proliferation, migration, and invasion as a

169 competing endogenous RNA of miR-129-5p[36]. In H9c2 cells, miR-129-5p inhibits autophagy and apoptosis induced

170 by hydrogen peroxide via the PI3K/AKT/mTOR signaling pathway[37]. So, we hypothesis TNRC6C-AS1 is an

171 important lncRNA in the regulation of PI3K-Akt signaling pathway in PTC's tumorigenesis.

172 LOC646736 is restricted expressed in thyroid. Our study is consisted with Xu et al's result[38]. The function of

173 LOC646736 in tumorigenesis is unknown. Further molecular biological experiments are required to confirm the

174 function of the identified genes.

175 In our study, we identified 4 pivotal lncRNAs in PTC. Among them, SLC26A4-AS1 is associated with PTC 176 disease free survival. It is meaningful to explore new biomarkers for diagnosis, prognostication, and therapeutic targets

177 of PTC so as to develop more effective surveillance and treatment programs. Though we had limited knowledge about

178 SLC26A4-AS1, this study provides a good direction of further study. Functional studies that further delineate the 179 biologic basis of the PTC are needed. Further studies with much larger sample sizes will be needed.

180

\section{Conclusion}

182 In conclusion, our data provide a comprehensive bioinformatics analysis of pivotal lncRNAs in PTC. These

184 potential indicators for prognosis.

185

186 Statements

187 Acknowledgements

$188 \quad$ Not applicable

189 Ethics approval and consent to participate

190 Not applicable

191 Competing interests

192 The authors declare that they have no competing interests

193 Funding

$194 \quad$ Not applicable 
195 Authors' contributions

196 FFS analyzed and interpreted the data. WWL was a major contributor in writing the manuscript. All authors read and 197 approved the final manuscript.

198

199

200

REFERENCE:

201 1. Hayes J, Peruzzi PP, Lawler S. MicroRNAs in cancer: biomarkers, functions and therapy. Trends Mol Med 2014;

20220 (8):460-469. doi:10.1016/j.molmed.2014.06.005

203 2. Slaby O, Laga R, Sedlacek O. Therapeutic targeting of non-coding RNAs in cancer. Biochem J 2017; 474 (24):4219-

204 4251. doi:10.1042/BCJ20170079

205 3. de Oliveira JC, Oliveira LC, Mathias C, Pedroso GA, Lemos DS, Salviano-Silva A, Jucoski TS, Lobo-Alves SC,

206 Zambalde EP, Cipolla GA, Gradia DF. LncRNAs in Cancer: another layer of complexity. J Gene Med; 2018 ; e3065.

207 doi:10.1002/jgm.3065

208 4. Salmena L, Poliseno L, Tay Y, Kats L, Pandolfi PP.A ceRNA hypothesis: the Rosetta Stone of a hidden RNA

209 language? Cell 2011;146 (3):353-358. doi:10.1016/j.cell.2011.07.014

210 5. de Oliveira JC, Oliveira LC, Mathias C, Pedroso GA, Lemos DS, Salviano-Silva A, Jucoski TS, Lobo-Alves SC,

211 Zambalde EP, Cipolla GA, Gradia DF. Long non-coding RNAs in cancer: Another layer of complexity. J Gene Med

212 2018; 21 (1):e3065. doi:10.1002/jgm.3065

213 6. Carling T, Udelsman R. Thyroid cancer. Annu Rev Med 2014; 65:125-137. doi:10.1146/annurev-med-061512214105739

215 7. Siegel RL, Miller KD, Jemal A. Cancer Statistics, 2017. CA Cancer J Clin 2017; 67 (1):7-30. 216 doi:10.3322/caac. 21387

217 8. Aschebrook-Kilfoy B, Schechter RB, Shih YC, Kaplan EL, Chiu BC, Angelos P, Grogan RH. The clinical and 218 economic burden of a sustained increase in thyroid cancer incidence. Cancer Epidemiol Biomarkers Prev 2013; 22

219 (7):1252-1259. doi:10.1158/1055-9965.EPI-13-0242

220 9. Nikiforov YE. Role of Molecular Markers in Thyroid Nodule Management: Then and Now. Endocrine practice :

221 official journal of the American College of Endocrinology and the American Association of Clinical Endocrinologists 222 2017; 23 (8):979-988. doi:10.4158/EP171805.RA

223 10. Lei H, Gao Y, Xu X. LncRNA TUG1 influences papillary thyroid cancer cell proliferation, migration and EMT

224 formation through targeting miR-145. Acta Biochim Biophys Sin (Shanghai) 2017; 49 (7):588-597. 
225

226

227

228

229

230

231

232

233

234

235

236

237

238

239

240

241

242

243

244

245

246

247

248

249

250

251

252

253

254

doi:10.1093/abbs/gmx047

11. Feng J, Zhou Q, Yi H, Ma S, Li D, Xu Y, Wang J, Yin S. A novel lncRNA n384546 promotes thyroid papillary cancer progression and metastasis by acting as a competing endogenous RNA of miR-145-5p to regulate AKT3. Cell Death Dis 2019; 10 (6):433. doi:10.1038/s41419-019-1637-7

12. Liang M, Jia J, Chen L, Wei B, Guan Q, Ding Z, Yu J, Rang R, He G. LncRNA MCM3AP-AS1 promotes proliferation and invasion through regulating miR-211-5p/SPARC axis in papillary thyroid cancer. Endocrine. 2019; doi:10.1007/s12020-019-01939-4

13. Love MI, Huber W, Anders S. Moderated estimation of fold change and dispersion for RNA-seq data with DESeq2. Genome Biol 2014; 15 (12):550. doi:10.1186/s13059-014-0550-8

14. Ritchie ME, Phipson B, Wu D, Hu Y, Law CW, Shi W, Smyth GK. limma powers differential expression analyses for RNA-sequencing and microarray studies. Nucleic Acids Res 2015; 43 (7):e47. doi:10.1093/nar/gkv007

15. Durinck S, Moreau Y, Kasprzyk A, Davis S, De Moor B, Brazma A, Huber W. BioMart and Bioconductor: a powerful link between biological databases and microarray data analysis. Bioinformatics 2005; 21 (16):3439-3440. doi:10.1093/bioinformatics/bti525

16. Paraskevopoulou MD, Vlachos IS, Karagkouni D, Georgakilas G, Kanellos I, Vergoulis T, Zagganas K, Tsanakas P, Floros E, Dalamagas T, Hatzigeorgiou AG. DIANA-LncBase v2: indexing microRNA targets on non-coding transcripts. Nucleic Acids Res 2016; 44 (D1):D231-238. doi:10.1093/nar/gkv1270

17. Li JH, Liu S, Zhou H, Qu LH, Yang JH. starBase v2.0: decoding miRNA-ceRNA, miRNA-ncRNA and proteinRNA interaction networks from large-scale CLIP-Seq data. Nucleic Acids Res 2014; 42 (Database issue):D92-97. doi:10.1093/nar/gkt1248

18. Yang JH, Li JH, Shao P, Zhou H, Chen YQ, Qu LH. starBase: a database for exploring microRNA-mRNA interaction maps from Argonaute CLIP-Seq and Degradome-Seq data. Nucleic Acids Res 2011 ; 39 (Database issue):D202-209. doi:10.1093/nar/gkq1056

19. Yi Y, Zhao Y, Li C, Zhang L, Huang H, Li Y, Liu L, Hou P, Cui T, Tan P, Hu Y, Zhang T, Huang Y, Li X, Yu J, Wang D. RAID v2.0: an updated resource of RNA-associated interactions across organisms. Nucleic Acids Res 2017; 45 (D1):D115-D118. doi:10.1093/nar/gkw1052

20. Karagkouni D, Paraskevopoulou MD, Chatzopoulos S, Vlachos IS, Tastsoglou S, Kanellos I, Papadimitriou D, Kavakiotis I, Maniou S, Skoufos G, Vergoulis T, Dalamagas T, Hatzigeorgiou AG. DIANA-TarBase v8: a decadelong collection of experimentally supported miRNA-gene interactions. Nucleic Acids Res 2018; 46 (D1):D239-D245. doi:10.1093/nar/gkx1141

PeerJ reviewing PDF | (2019:04:36581:1:1:NEW 4 Jul 2019) 
255 21. Agarwal V, Bell GW, Nam JW, Bartel DP. Predicting effective microRNA target sites in mammalian mRNAs.

256 Elife 4. 2015; doi:10.7554/eLife.05005

257 22. Shannon P, Markiel A, Ozier O, Baliga NS, Wang JT, Ramage D, Amin N, Schwikowski B, Ideker T. Cytoscape:

258 a software environment for integrated models of biomolecular interaction networks. Genome Res 2003; 13 (11):2498-

259 2504. doi:10.1101/gr.1239303

260 23. Kanehisa M, Furumichi M, Tanabe M, Sato Y, Morishima K. KEGG: new perspectives on genomes, pathways,

261 diseases and drugs. Nucleic Acids Res 2017; 45 (D1):D353-D361. doi:10.1093/nar/gkw1092

262 24. Szklarczyk D, Morris JH, Cook H, Kuhn M, Wyder S, Simonovic M, Santos A, Doncheva NT, Roth A, Bork P,

263 Jensen LJ, von Mering C. The STRING database in 2017: quality-controlled protein-protein association networks,

264 made broadly accessible. Nucleic Acids Res 2017; 45 (D1):D362-D368. doi:10.1093/nar/gkw937

265 25. Bindea G, Mlecnik B, Hackl H, Charoentong P, Tosolini M, Kirilovsky A, Fridman WH, Pagès F, Trajanoski Z,

266 Galon J. ClueGO: a Cytoscape plug-in to decipher functionally grouped gene ontology and pathway annotation

267 networks. Bioinformatics 2009; 25 (8):1091-1093. doi:10.1093/bioinformatics/btp101

268 26. Bindea G, Galon J, Mlecnik B. CluePedia Cytoscape plugin: pathway insights using integrated experimental and

269 in silico data. Bioinformatics 2013; 29 (5):661-663. doi:10.1093/bioinformatics/btt019

270 27. Tang Z, Li C, Kang B, Gao G, Li C, Zhang Z. GEPIA: a web server for cancer and normal gene expression

271 profiling and interactive analyses. Nucleic Acids Res 2017; 45 (W1):W98-W102. doi:10.1093/nar/gkx247

272 28. Cancer Genome Atlas Research N. Integrated genomic characterization of papillary thyroid carcinoma. Cell 2014;

273159 (3):676-690. doi:10.1016/j.cell.2014.09.050

274 29. Hao RT, Zheng C, Wu CY, Xia EJ, Zhou XF, Quan RD, Zhang XH. NECTIN4 promotes papillary thyroid cancer

275 cell proliferation, migration, and invasion and triggers EMT by activating AKT. Cancer Manag Res 2019; 11:2565-

276 2578. doi:10.2147/CMAR.S190332

277 30. Zheng Z, Zhou X, Cai Y, Chen E, Zhang X, Wang O, Wang Q, Liu H. TEKT4 Promotes Papillary Thyroid Cancer

278 Cell Proliferation, Colony Formation, and Metastasis through Activating PI3K/Akt Pathway. Endocrine pathology

279 2018; 29 (4):310-316. doi:10.1007/s12022-018-9549-0

280 31. Wen J, Wang H, Dong T, Gan P, Fang H, Wu S, Li J, Zhang Y, Du R, Zhu Q. STAT3-induced upregulation of

281 lncRNA ABHD11-AS1 promotes tumour progression in papillary thyroid carcinoma by regulating miR-1301-

282 3p/STAT3 axis and PI3K/AKT signalling pathway. Cell Prolif 2019; 52 (2):e12569. doi:10.1111/cpr.12569

283 32. Liu C, Feng Z, Chen T, Lv J, Liu P, Jia L, Zhu J, Chen F, Yang C, Deng Z. Downregulation of NEAT1 reverses

284 the radioactive iodine resistance of papillary thyroid carcinoma cell via miR-101-3p/FN1/PI3K-AKT signaling

Peer) reviewing PDF | (2019:04:36581:1:1:NEW 4 Jul 2019) 
285 pathway. Cell Cycle 2019; 18 (2):167-203. doi:10.1080/15384101.2018.1560203

286 33. Fagerberg L, Hallstrom BM, Oksvold P, Kampf C, Djureinovic D, Odeberg J, Habuka M, Tahmasebpoor S, 287 Danielsson A, Edlund K, Asplund A, Sjöstedt E, Lundberg E, Szigyarto CA, Skogs M, Takanen JO, Berling H, Tegel

288 H, Mulder J, Nilsson P, Schwenk JM, Lindskog C, Danielsson F, Mardinoglu A, Sivertsson A, von Feilitzen K,

289 Forsberg M, Zwahlen M, Olsson I, Navani S, Huss M, Nielsen J, Ponten F, Uhlén M. Analysis of the human tissue-

290 specific expression by genome-wide integration of transcriptomics and antibody-based proteomics. Mol Cell

291 Proteomics 2014; 13 (2):397-406. doi:10.1074/mcp.M113.035600

292 34. Huang H, Chen J, Ding CM, Jin X, Jia ZM, Peng J. LncRNA NR2F1-AS1 regulates hepatocellular carcinoma 293 oxaliplatin resistance by targeting ABCC1 via miR-363. J Cell Mol Med 2018; 22 (6):3238-3245. 294 doi:10.1111/jcmm.13605

295 35. Wang L, Zhao S, Mingxin YU. LncRNA NR2F1-AS1 is involved in the progression of endometrial cancer by 296 sponging miR-363 to target SOX4. Pharmazie 2019; 74 (5):295-300. doi:10.1691/ph.2019.8905

297 36. Hou S, Lin Q, Guan F, Lin C. LncRNA TNRC6C-AS1 regulates UNC5B in thyroid cancer to influence cell 298 proliferation, migration, and invasion as a competing endogenous RNA of miR-129-5p. J Cell Biochem 2018 ; 119 299 (10):8304-8316. doi:10.1002/jcb.26868

300 37. Zhang H, Zhang X, Zhang J. MiR-129-5p inhibits autophagy and apoptosis of H9c2 cells induced by hydrogen

301 peroxide via the PI3K/AKT/mTOR signaling pathway by targeting ATG14. Biochem Biophys Res Commun 2018;

302506 (1):272-277. doi:10.1016/j.bbrc.2018.10.085

303 38. Xu Y, Deng Y, Ji Z, Liu H, Liu Y, Peng H, Wu J, Fan J. Identification of thyroid carcinoma related genes with 304 mRMR and shortest path approaches. PLoS One 2014; 9 (4):e94022. doi:10.1371/journal.pone.0094022

305

306 Figure Legends

307 Fig. 1. Distribution analysis of gene expression. (A-C): Distribution of gene expression levels in each group, where 308 the $\mathrm{x}$-axis indicates the $\log 2$ value $(\mathrm{TPM}+1)$ and the $\mathrm{y}$-axis shows the proportion of genes. (D-F): Principle component 309 analysis of each sample. Dots represent the PC value of each sample. (G-I): Distribution of genes based on the

310 expression level of each sample, where $\mathrm{x}$-axis shows each analyzed sample and y-axis shows the gene value 311 distribution in each sample.

312 Fig. 2. (A-C) Volcano graph displaying pairs of expressed genes. Red dots indicate significantly up-regulated 313 differential expressed genes and blue dots indicate significantly down-regulated DEGs that passed the screening 314 threshold. (D) differential expressed gene counts of each dataset. (E) Venn analysis among DEGs of 3 datastes. (F) 
315 Heatmap and hierarchical clustering of identified DEGs. Up or down regulated genes are colored in red or blue.

316 Fig. 3. LncRNA-miRNA-mRNA ceRNA network in PTC. Hexagon represented cancer specific lncRNA. Octagon

317 represented cancer specific miRNA. Ellipse represented cancer specific mRNA.

318 Fig. 4. (A-C) Gene ontology analysis of mRNAs in ceRNA network. X-axis reflects gene count; Y-axis reflects

319 different GO terms. The column color reflects $\mathrm{p}$ value $(-\log 10(\mathrm{p}$ value $))$ : black represents the biggest value, blue 320 represents the smallest value.

321 Fig. 5. Significantly enriched pathway terms of mRNAs in ceRNA network. The node size reflects gene count: the 322 bigger the gene count, the bigger the node size is. The node color reflects $p$ value $[-\log 10(p$ value $)]:$ the bigger the $323 \log 10$ ( $\mathrm{p}$ value) value, the darker the node color is

324 Fig. 6. (A), (B) and (C): Analyses of pivotal lncRNAs expression in tumor vs normal tissues and in different tumor 325 stages. Red box stands for tumor tissue; grey box is for normal tissue; and dots presents each sample value in left 326 panel. ${ }^{*} \mathrm{P}<0.05$; other $\mathrm{P}$-values are shown on the diagrams. (D), (E), and (F): Overall and disease-free survival 327 analyses of hub lncRNAs. P-values are shown on the diagrams.

328

329

330 


\section{Figure $\mathbf{1}$ (on next page)}

Figure 1 Distribution analysis of gene expression.

Fig. 1. Distribution analysis of gene expression. (A-C): Distribution of gene expression levels in each group, where the $x$-axis indicates the log2 value $(T P M+1)$ and the $y$-axis shows the proportion of genes. (D-F): Principle component analysis of each sample. Dots represent the PC value of each sample. (G-I): Distribution of genes based on the expression level of each sample, where $x$-axis shows each analyzed sample and $y$-axis shows the gene value distribution in each sample. 
A

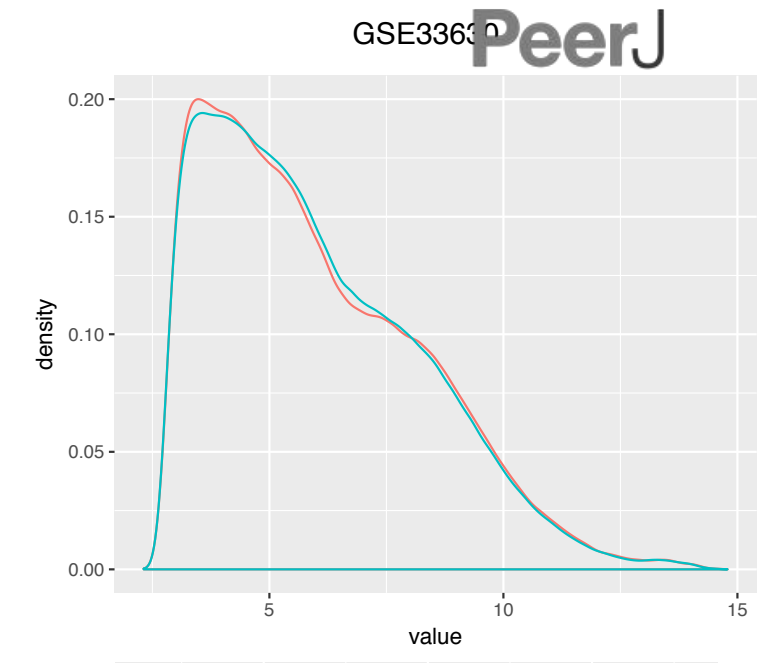

D

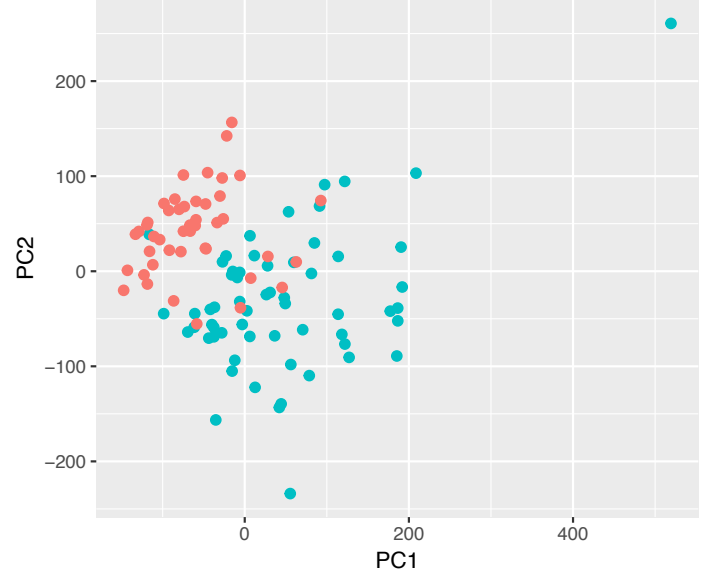

B

GSE3467 Manuscript todbe reviewed GSE3678
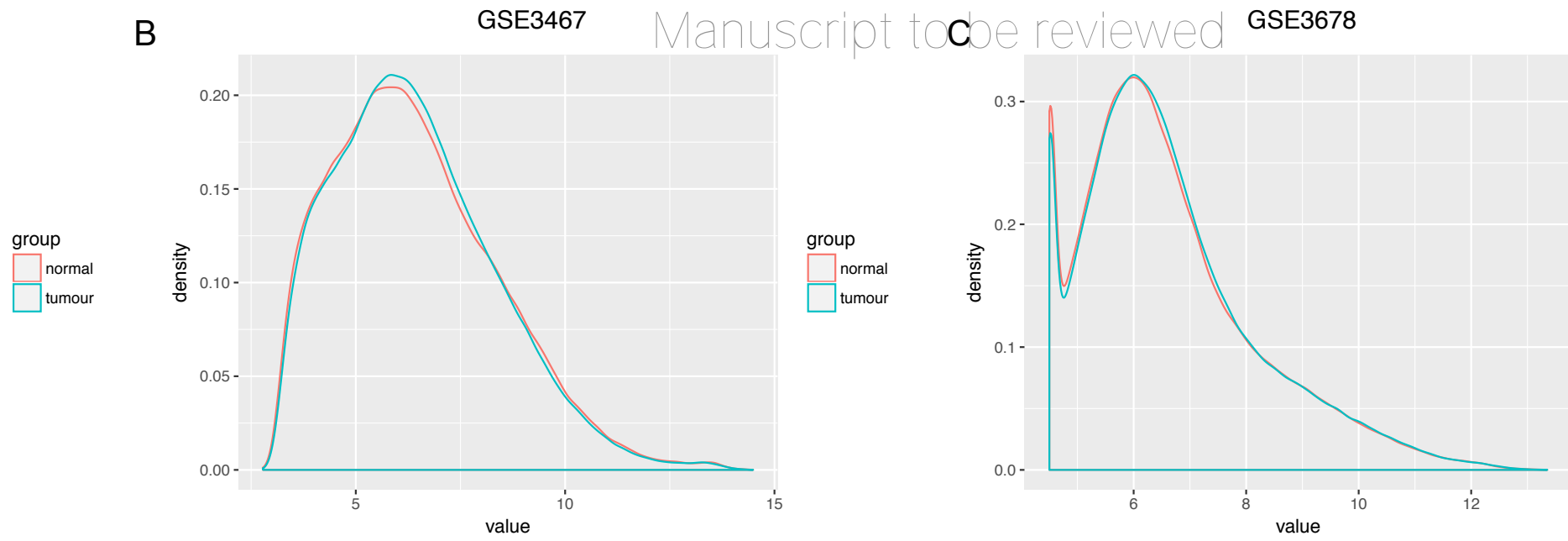

group
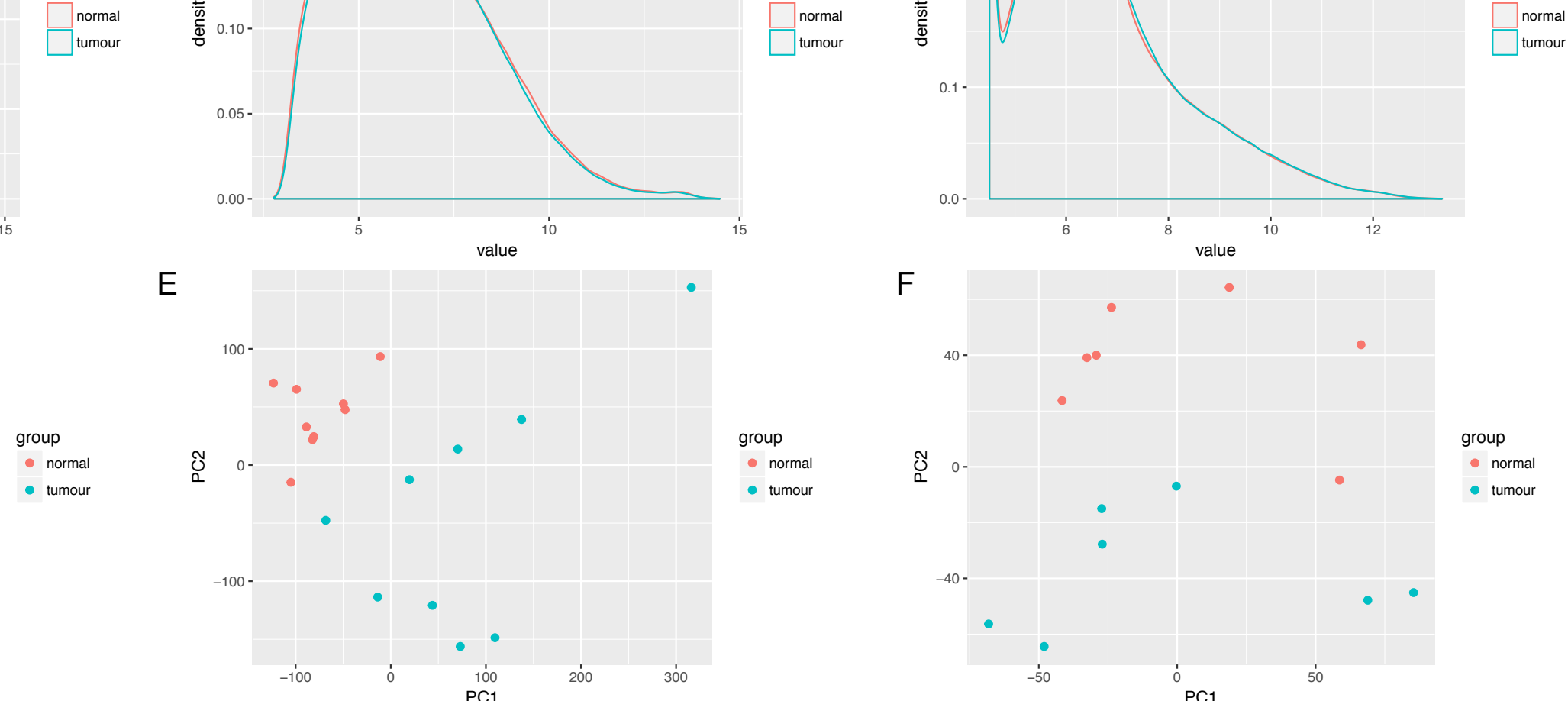

E

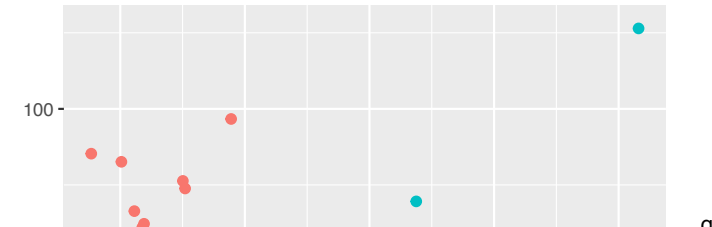

$\operatorname{som}_{0}$

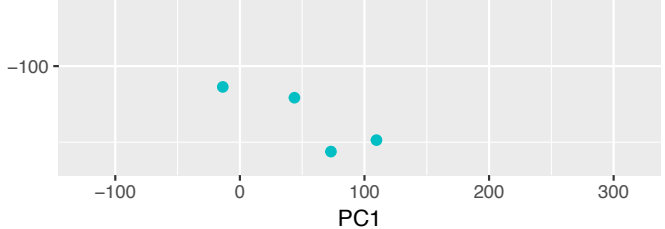

$\mathrm{F}$

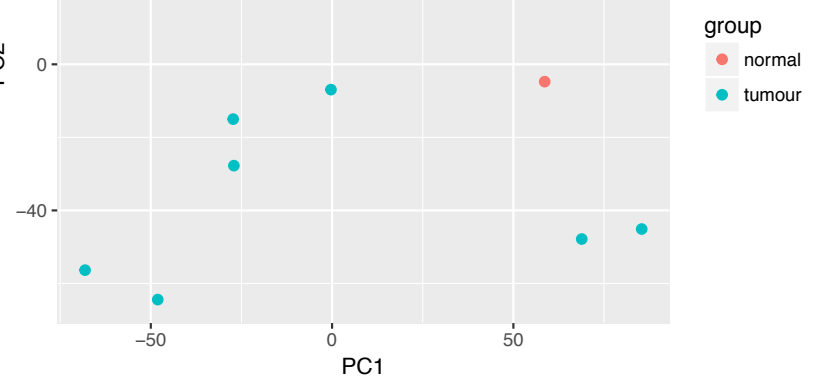

G

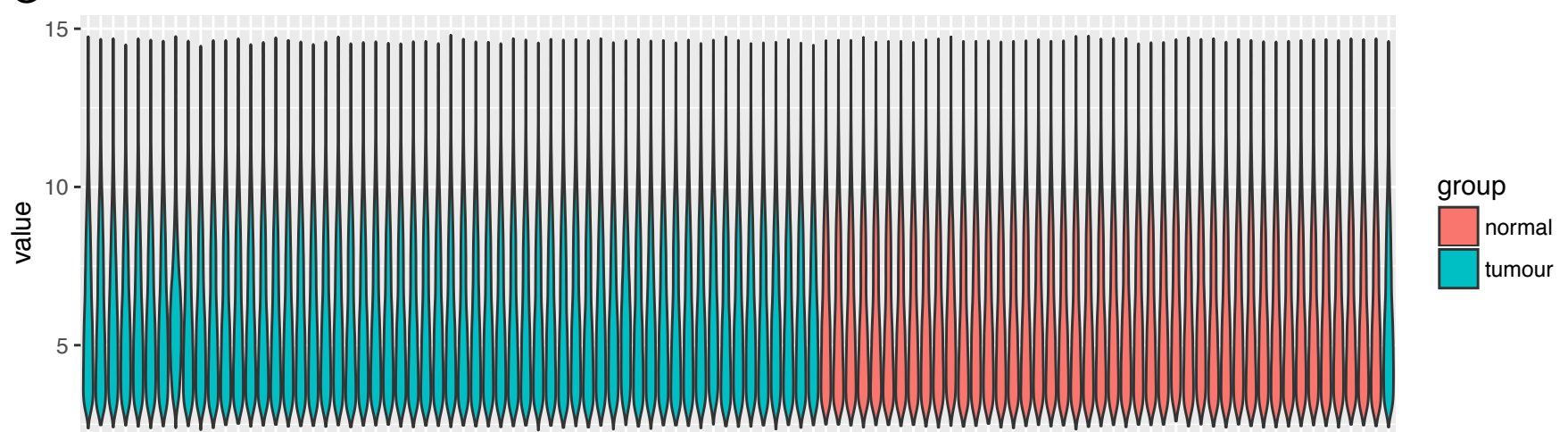

samples in GSE33630

$\mathrm{H}$

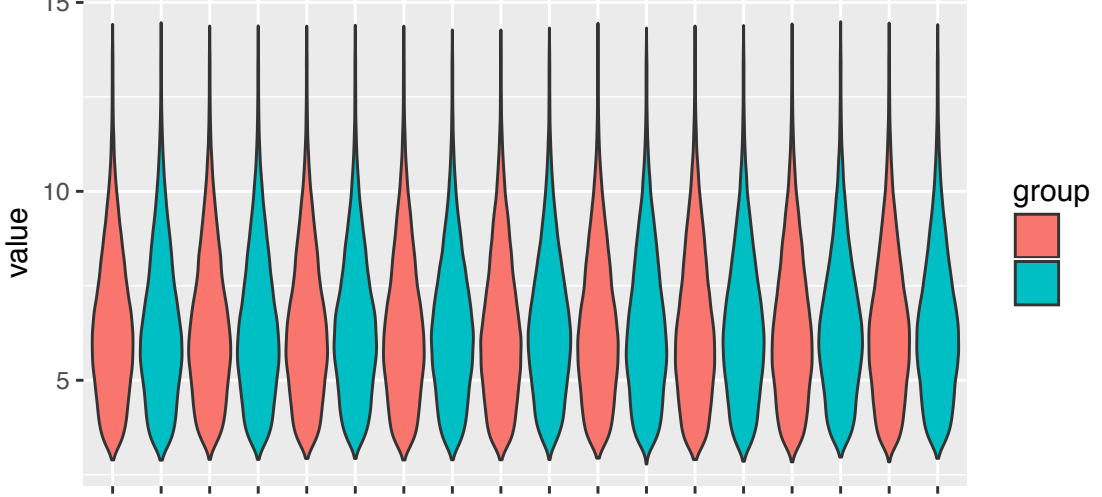

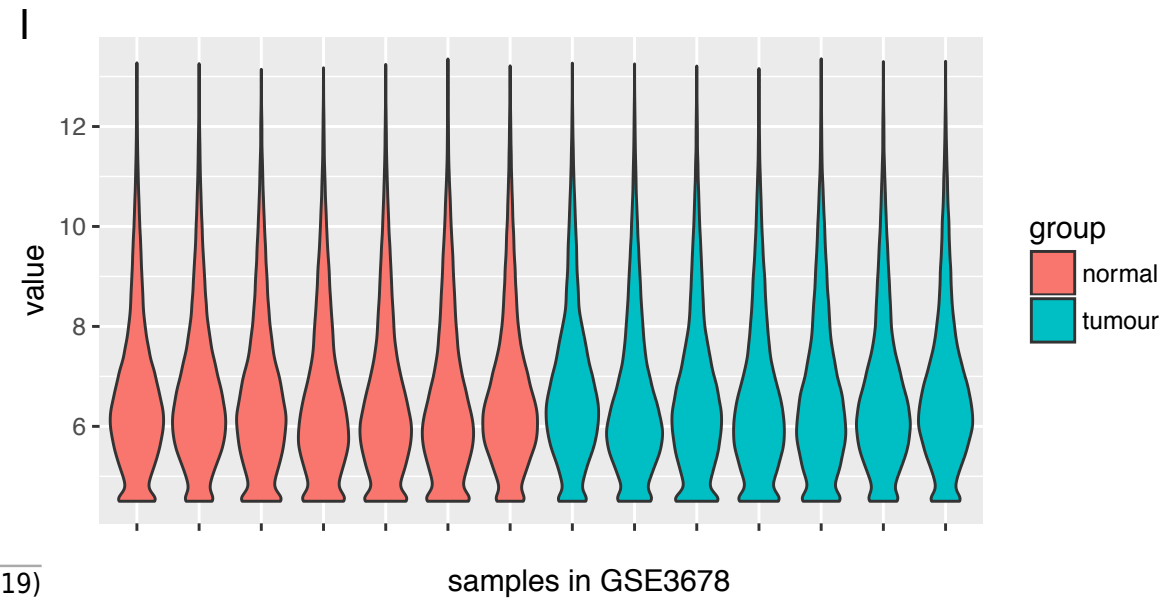




\section{Figure 2}

Fig. 2. gene expression of each dataset.

Fig. 2. (A-C) Volcano graph displaying pairs of expressed genes. Red dots indicate significantly up-regulated differential expressed genes and blue dots indicate significantly down-regulated DEGs that passed the screening threshold. (D) differential expressed gene counts of each dataset. (E) Venn analysis among DEGs of 3 datastes. (F) Heatmap and hierarchical clustering of identified DEGs. Up or down regulated genes are colored in red or blue. 
A

GSE33630 The number of up gene is 1112
The number of down gene is 1114

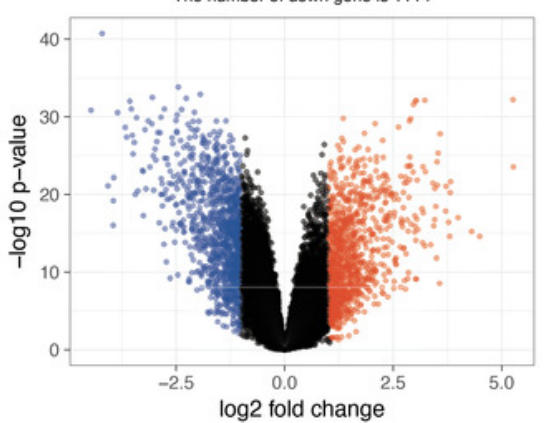

B

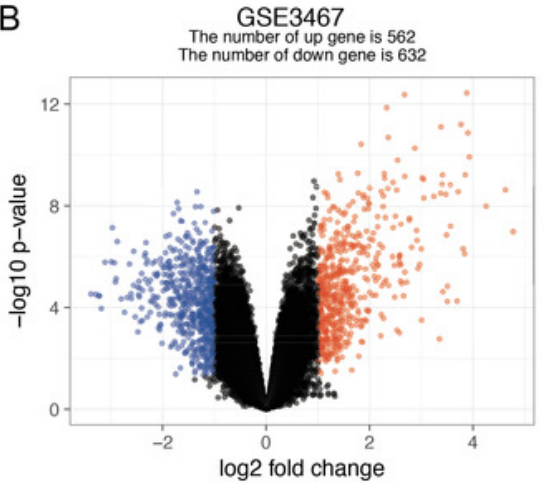

C

GSE3678

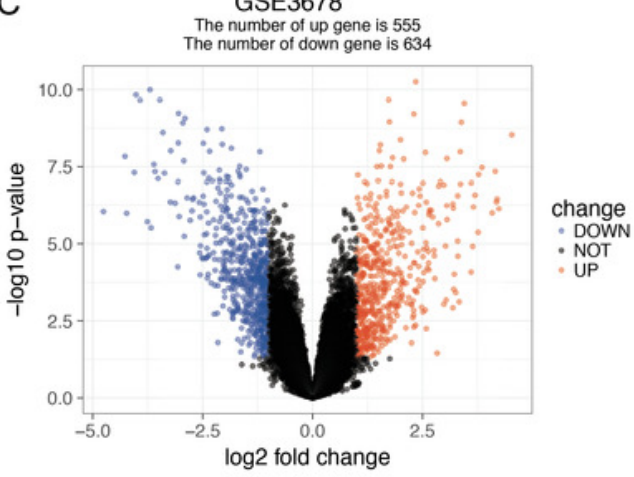

change DOWN : NOT

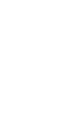

Change
chown
:NOT
D

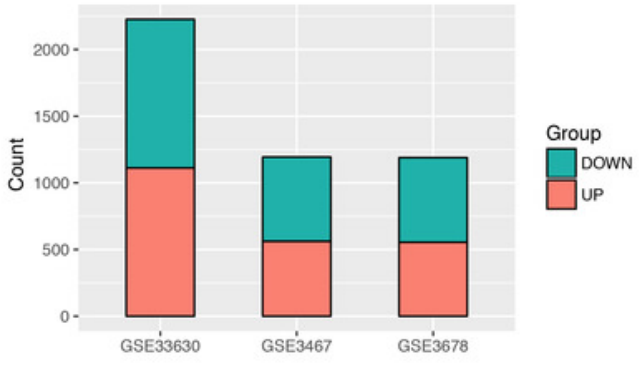

E

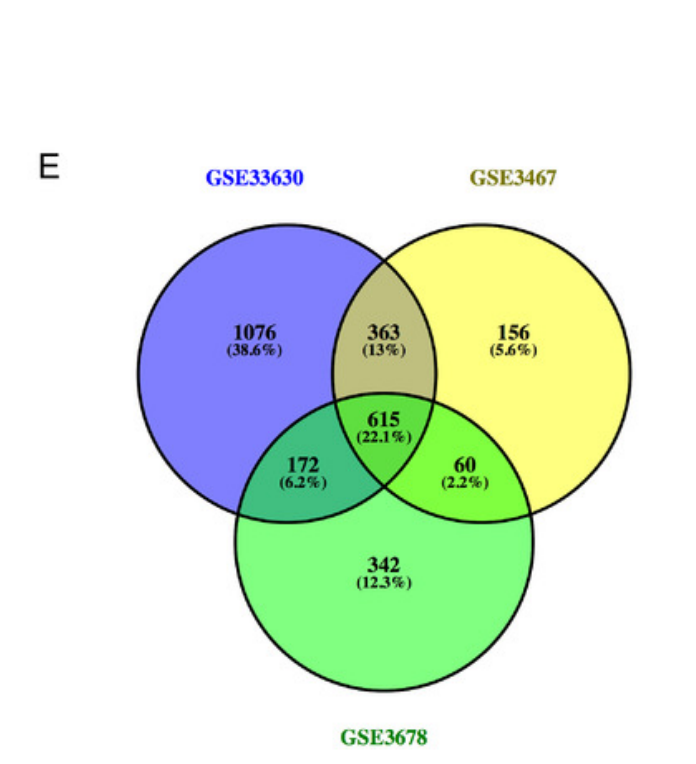

GSE3678
F

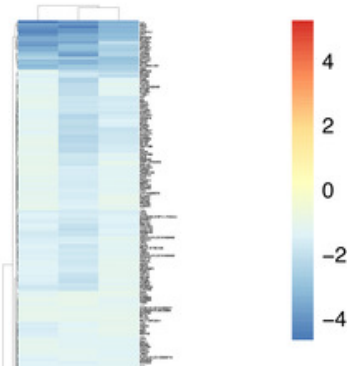


Figure 3 (on next page)

Fig. 3. LncRNA-miRNA-mRNA ceRNA network in PTC.

Fig. 3. LncRNA-miRNA-mRNA ceRNA network in PTC. Hexagon represented cancer specific IncRNA. Octagon represented cancer specific miRNA. Ellipse represented cancer specific mRNA. 


\section{PeerJ}

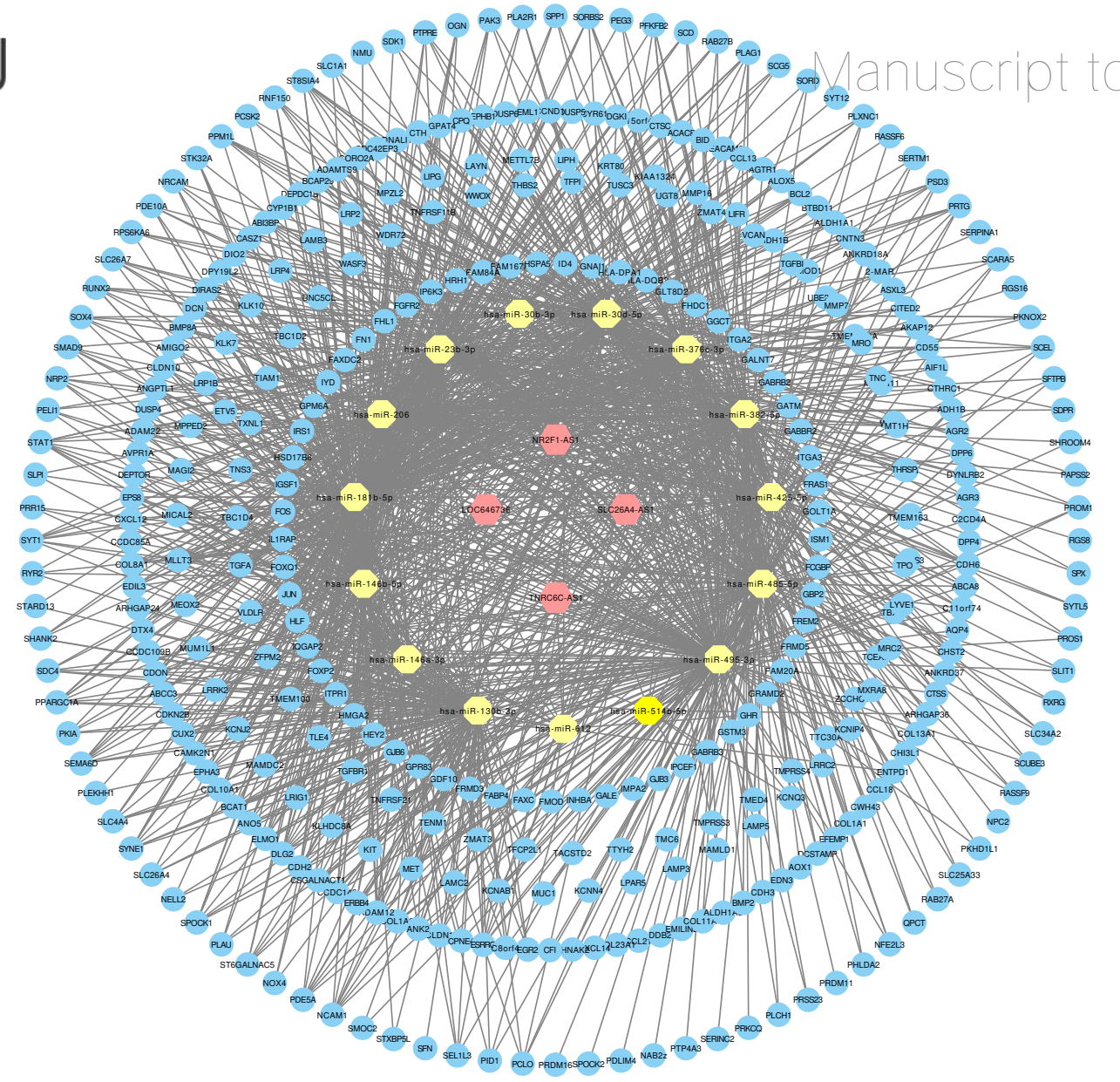


Figure 4 (on next page)

Figure 4 Gene ontology analysis of mRNAs in ceRNA network.

Fig. 4. (A-C) Gene ontology analysis of mRNAs in ceRNA network. X-axis reflects gene count; Y-axis reflects different GO terms. The column color reflects $p$ value (- $\log 10$ ( $p$ value)): black represents the biggest value, blue represents the smallest value. 
A

positive regulation of cell migration -

blood coagulation osteoblast differentiation Peerjorvous system development angiogenesis cell proliferation immune response response to drug inflammatory response positive regulation of gene expression proteolysis positive regulation of GTPase activity positive regulation of cell proliferation extracellular matrix organization cell adhesion -

B

integral component of plasma membrane extracellular region extracellular space extracellular exosome plasma membrane integral component of membrane perinuclear region of cytoplasm proteinaceous extracellular matrix endoplasmic reticulum extracellular matrix cell surface cell junction focal adhesion apical plasma membrane -

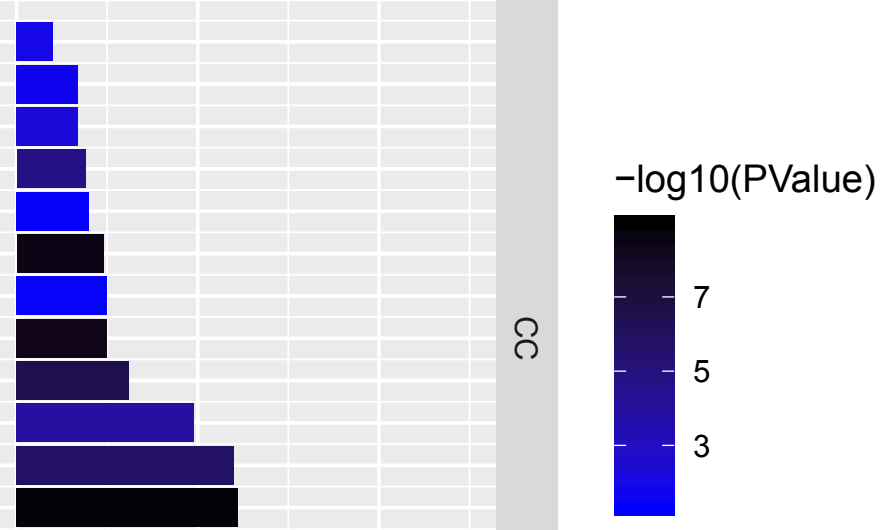

C

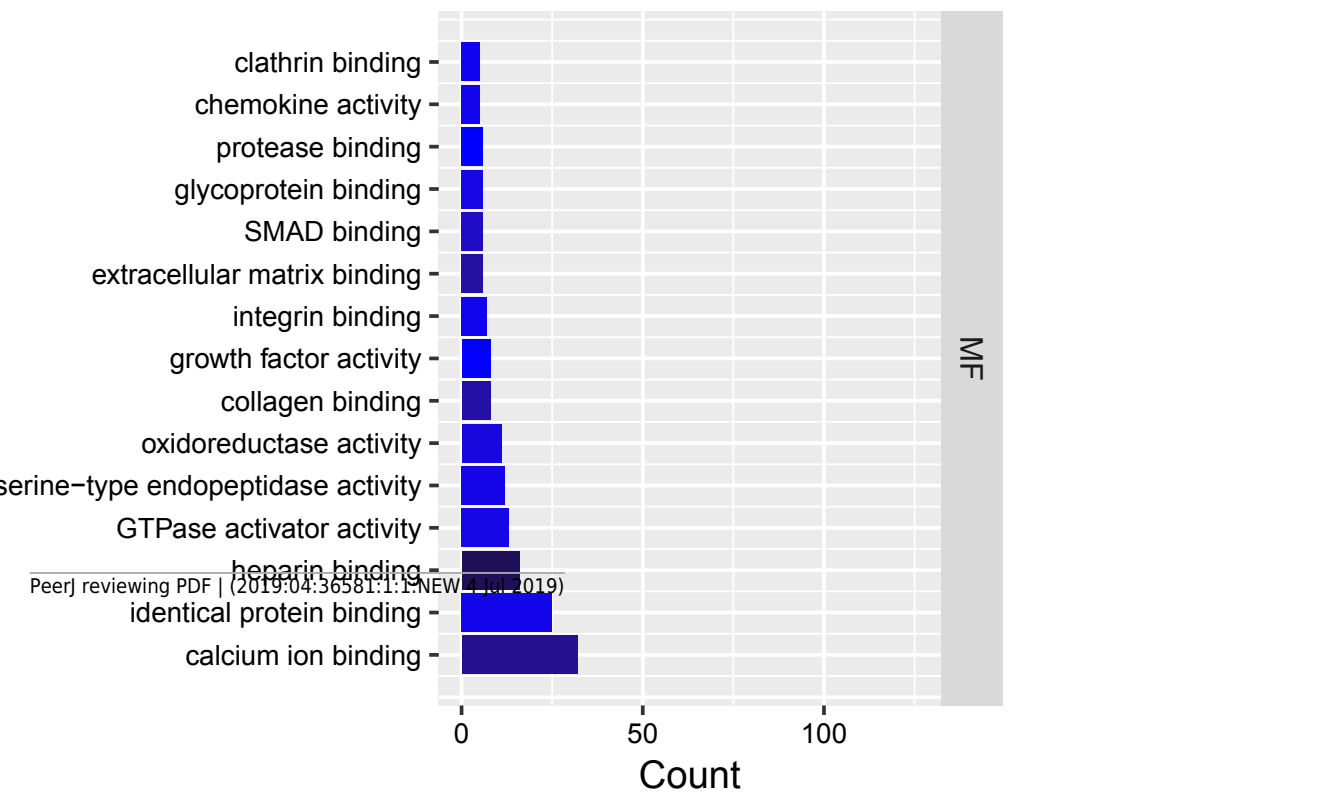


Figure $\mathbf{5}$ (on next page)

Figure 5 Significantly enriched pathway terms of mRNAs in ceRNA network.

Fig. 5. Significantly enriched pathway terms of mRNAs in ceRNA network. The node size reflects gene count: the bigger the gene count, the bigger the node size is. The node color reflects $p$ value $[-\log 10(p$ value $)]$ : the bigger the $-\log 10(p$ value) value, the darker the node color is 
Cytokine-cytokine receptor interaction -

Proteoglycans in cancer -

\section{Pathways in cancer -}

Cell adhesion molecules (CAMs) -

Axon guidance -

Focal adhesion -

Thyroid hormone synthesis -

Complement and coagulation cascades -

Protein digestion and absorption -

Small cell lung cancer-

TGF-beta signaling pathway -

ECM-receptor interaction -

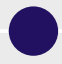

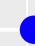

-

$\bullet$

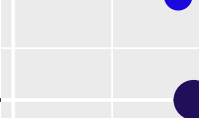

Count

- 10

- 15

20

-log10(PValue)

-4.0
-3.5
-3.0
-2.5
-2.0


Figure 6 (on next page)

Fig. 6. (A), (B) and (C): Analyses of pivotal IncRNAs expression in tumor vs normal tissues and in different tumor stages. (D), (E), and (F): Overall and disease-free survival analyses of hub IncRNAs.

Fig. 6. (A), (B) and (C): Analyses of pivotal IncRNAs expression in tumor vs normal tissues and in different tumor stages. Red box stands for tumor tissue; grey box is for normal tissue; and dots presents each sample value in left panel. $* \mathrm{P}<0.05$; other $\mathrm{P}$-values are shown on the diagrams. (D), (E), and (F): Overall and disease-free survival analyses of hub IncRNAs. Pvalues are shown on the diagrams. 


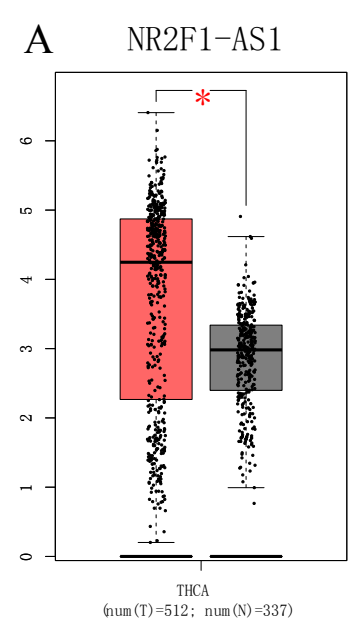

Peerd2F1-AS1

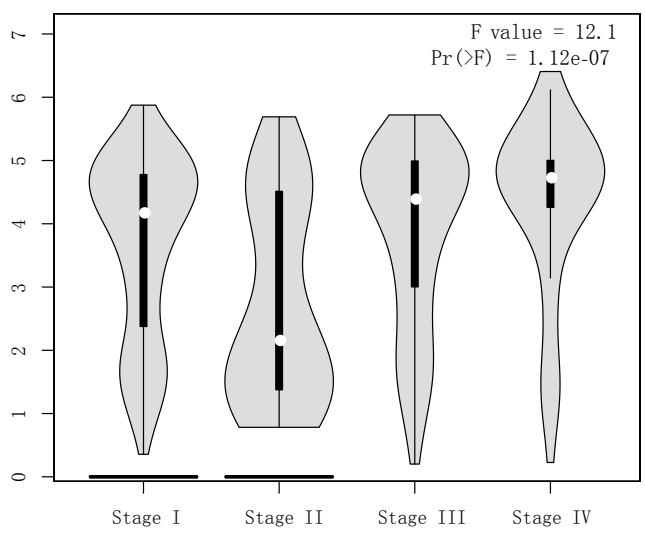

B SLC26A4-AS1

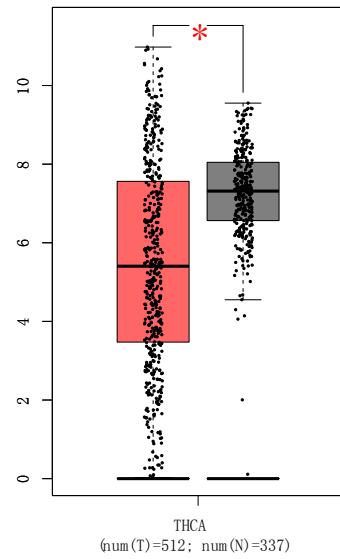

C TNRC6C-AS1

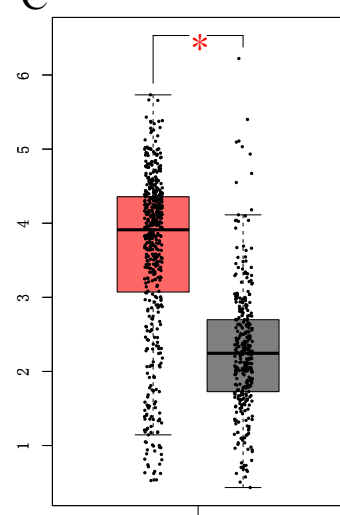

$\underset{\operatorname{mum}(\mathrm{T})=512 ; \operatorname{num}(\mathrm{N})=337)}{\text { THCA }}$
SLC26A4-AS1

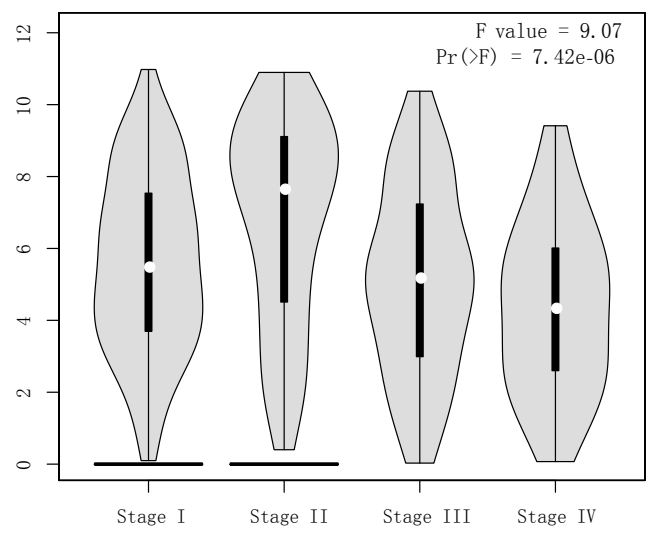

TNRC6C-AS1

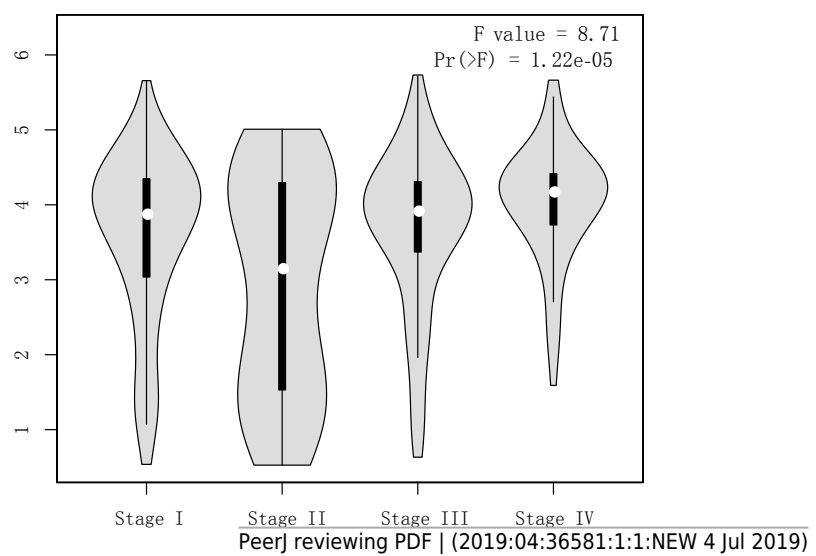

D

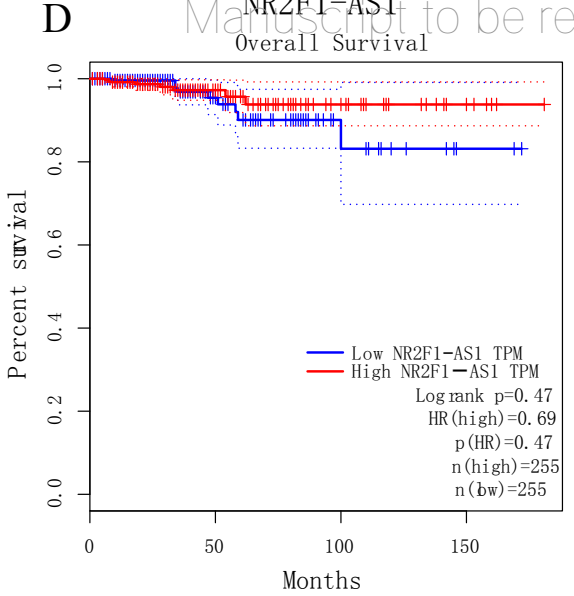

wed

NR2F1-AS1

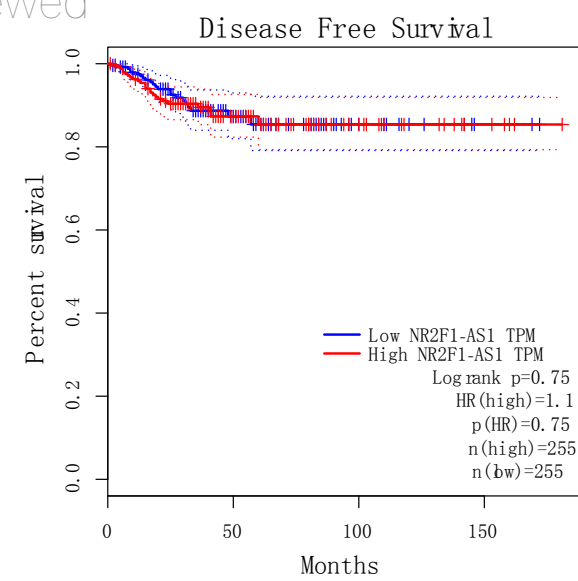

SLC26A4-AS1
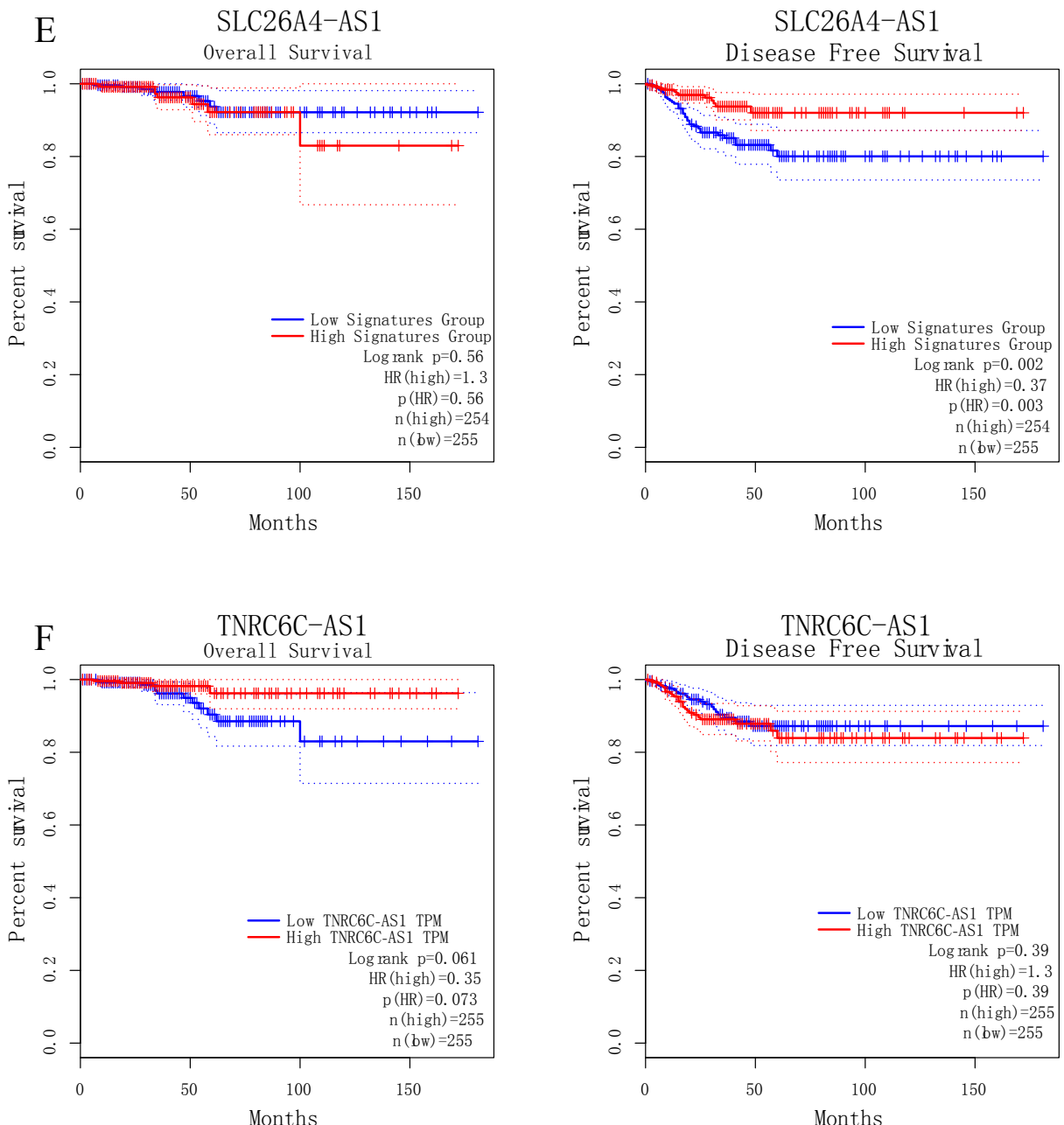